\title{
A Geometric Application of Nori's Connectivity Theorem
}

\author{
CLAIRE VOISIN
}

\begin{abstract}
We study (rational) sweeping out of general hypersurfaces by varieties having small moduli spaces.

As a consequence, we show that general $K$-trivial hypersurfaces are not rationally swept out by abelian varieties of dimension at least two.

As a corollary, we show that Clemens' conjecture on the finiteness of rational curves of given degree in a general quintic threefold, and Lang's conjecture saying that such varieties should be rationally swept-out by abelian varieties, contradict.
\end{abstract}

Mathematics Subject Classification (2000): 14C05 (primary); 14D07 (secondary).

\section{0. - Introduction}

Our purpose in this paper is to contribute to the study of rational maps from $r$-dimensional varieties to general hypersurfaces in projective space (cf [5], [20], [12], [4]). In the last section, we shall eventually extend this to the study of correspondences instead of rational maps. The problem we consider is the following: given a family $\mathcal{Y} \rightarrow \mathcal{S}$ of $r$-dimensional smooth projective varieties, when is a general hypersurface $X$ of degree d in projective space $\mathbb{P}^{n+1}$ swept out by images of rational maps from one member of this family to $X$ ?

(Recall that the word "general" in this context means "away from countably many proper Zariski closed subsets of the moduli space”.)

Our approach to this problem is Hodge theoretic. Unlike [5], [20], [12], [4], the result has nothing to do with the canonical bundle of the varieties $Y_{t}, t \in \mathcal{S}$. Instead, our answer will depend only on the dimension of the moduli space $\mathcal{S}$. Roughly speaking, the idea is as follows: assume that $\operatorname{dim} \mathcal{S}$ is small, but the general $X$ is covered by images of rational maps from one member of this family to $X$; then there is a universal dominating rational map $\Phi$ fitting in the following commutative diagram

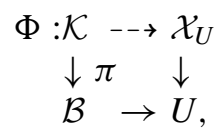

Pervenuto alla Redazione l'1 settembre 2003 e in forma definitiva il 6 luglio 2004. 
where $\pi: \mathcal{K} \rightarrow \mathcal{B}$ is induced by the family $\mathcal{Y} \rightarrow \mathcal{S}$ via a certain map $\mathcal{B} \rightarrow$ $\mathcal{S}$, and $\mathcal{X}_{U} \rightarrow U$ is the universal family of hypersurfaces of degree $d$ in $\mathbb{P}^{n+1}$. Here $U$ is the open set of $\mathbb{P}\left(H^{0}\left(\mathbb{P}^{n+1}, \mathcal{O}_{\mathbb{P}^{n+1}}(d)\right)\right)$ parameterizing smooth hypersurfaces.

If we fix a point $s \in \mathcal{S}$, we have now $\mathcal{B}_{s}$, which is the fiber of the map $\mathcal{B} \rightarrow \mathcal{S}$, and $\mathcal{X}_{\mathcal{B}_{s}}$, which is the fibered product

$$
\mathcal{B}_{s} \times_{U} \mathcal{X} \text {. }
$$

The $\Phi$ above restricts to

$$
\Phi_{s}: Y_{s} \times \mathcal{B}_{s} \rightarrow \mathcal{X}_{\mathcal{B}_{s}}
$$

The point now is that if $\operatorname{dim} \mathcal{S}$ is small, then the corank of the map $\mathcal{B}_{s} \rightarrow U$ is small, so that an adaptation of Nori's connectivity theorem [18] will show that the cohomology groups of $\mathcal{X}_{\mathcal{B}_{s}}$, modulo the cohomology of $\mathbb{P}^{n+1} \times \mathcal{B}_{s}$, are 0 or have small Hodge level. Studying the cohomology class of the graph of $\Phi$ in

$$
H^{2 n}\left(\mathcal{K} \times_{\mathcal{B}} \mathcal{X}_{\mathcal{B}}, \mathbb{Q}\right)
$$

via the Leray spectral sequence of the map

$$
\mathcal{K} \times_{\mathcal{B}} \mathcal{X}_{\mathcal{B}} \rightarrow \mathcal{S}
$$

with fiber $Y_{S} \times \mathcal{X}_{\mathcal{B}_{s}}$, we will deduce a contradiction with the fact that our initial $\Phi$ was dominating.

Let us now state our precise results:

Definition 1. Let $\mathcal{Y} \rightarrow \mathcal{S}$ be a family of $r$-dimensional smooth projective varieties. We say that a $n$-dimensional variety $X$ is rationally swept out by varieties parametrized by $\mathcal{S}$, if there exist a quasiprojective variety $B$ of dimension $n-r$, a family $K \rightarrow B$ which is the pull-back of the family $\mathcal{Y}$ via a morphism $\psi: B \rightarrow \mathcal{S}$, and a dominant rational map

$$
\phi: K \rightarrow X \text {, }
$$

(which is necessarily generically finite on the generic fiber $K_{b}$ since $\operatorname{dim} K=$ $\operatorname{dim} X)$.

REMARK 1. One can show that this property is equivalent to the fact that the union of the images of generically finite rational maps from a variety $Y_{t}, t \in \mathcal{S}$, to $X$ contains a Zariski open set of $X$.

With this definition, we show:

THEOREM 1. Fix an integer $1 \leq r \leq n$. Let $\gamma=\frac{r-1}{2}$, $r$ odd, or $\gamma=\frac{r}{2}, r$ even, that is $\gamma$ is the round-up of $\frac{r-1}{2}$. Let $\mathcal{Y} \rightarrow \mathcal{S}, \operatorname{dim} \mathcal{S}=C$, be a family of $r$-dimensional smooth projective varieties. Then the general hypersurface of degree $d$ in $\mathbb{P}^{n+1}$ is not rationally swept out by varieties parameterized by $\mathcal{S}$ if

$$
\begin{aligned}
& (d+1) r \geq 2 n+C+2, \\
& (\gamma+1) d \geq 2 n-r+1+C .
\end{aligned}
$$

(Note that except for $r=1,2$, the second inequality implies the first.) 
REMARK 2. One could of course prove a similar statement for sufficiently ample hypersurfaces in any smooth variety. In the case of projective space, the estimates on $d$ are sharp, and allow applications to the Calabi-Yau case, which is never considered in the papers quoted above (see Section 3).

So let us consider the case of Calabi-Yau hypersurfaces. Because of the Lang conjecture that we shall recall below, we will be mainly interested into the case where the family $\mathcal{Y} \rightarrow \mathcal{S}$ is a family of abelian varieties. Our Theorem 1 implies in this case:

THEOREM 2. Let $X$ be a general Calabi-Yau hypersurface in projective space $\mathbb{P}^{n+1}$, that is $d=n+2$. Then $X$ is not rationally swept out by $r$-dimensional abelian varieties, for any $r \geq 2$.

In the paper [16], Lang formulates a number of conjectures concerning smooth projective complex varieties $X$. One of them is that the analytic closure of the union of the images of holomorphic maps from $\mathbb{C}$ to $X$ is equal to the union of the images of non constant rational maps from an abelian variety to $X$. Another one is that this locus is equal to $X$ itself if and only if $X$ is not of general type.

Next, by a standard countability argument for Chow varieties (similar to the one given in the beginning of Section 2), we see that, according to these conjectures, if $X$ is not of general type, there should exist a quasiprojective variety $B$, a family $K \rightarrow B$ of abelian varieties, and a dominating rational map

$$
\phi: K \rightarrow X,
$$

which is non constant on the generic fiber $K_{b}, b \in B$.

Let us now consider the case where $X$ is a Calabi-Yau variety, that is $K_{X}$ is trivial. We claim that if a map $\phi$ as above exists, then we may assume that $\phi_{\mid K_{b}}$ is generically finite, for generic $b \in B$. Indeed, because $H^{0}\left(X, K_{X}\right) \neq 0$, for generic $b \in B$, the image $\phi\left(K_{b}\right)$ has effective canonical bundle, in the sense that any desingularization $Z_{b}$ of it has effective canonical bundle, as follows from adjunction formula and the fact that the $\phi\left(K_{b}\right)$ cover $X$. Now it is immediate to prove that any dominant rational map

$$
K_{b} \rightarrow Z_{b}
$$

where $K_{b}$ is an abelian variety and $Z_{b}$ has effective canonical bundle, factors through some quotient map $K_{b} \rightarrow K_{b}^{\prime}$, where $K_{b}^{\prime}$ is an abelian variety which is a quotient of $K_{b}$, and has the same dimension as $Z_{b}$. Replacing the family of abelian varieties $\left(K_{b}\right)_{b \in B}$, by the family $\left(K_{b}^{\prime}\right)_{b \in B}$ gives the desired $\phi^{\prime}$.

In other words, Lang's conjecture asserts in particular that a Calabi-Yau variety should be rationally swept out by $r$-dimensional abelian varieties, for some $r \geq 1$. Hence, if Lang's conjecture is true, such an $X$ should be swept out by elliptic curves. 
On the other hand, the following is proved in [7], lecture 22:

Proposition 1. If a general Calabi-Yau hypersurface of dimension $\geq 2$ is rationally swept-out by elliptic curves, then it contains a divisor which is uniruled.

In dimension 3, this proposition combined with Theorem 2 shows that Lang's conjecture and Clemens' conjecture (see [6]) on the finiteness of rational curves of fixed degree in a general quintic threefold, contradict.

In the case of hypersurfaces of general type, inequality $(0.1)$ can be applied to give a non trivial estimate on the minimal genus of covering families of curves, but the estimate is not sharp and could be obtained directly by geometry. What is interesting however is that looking more precisely at the proof of Theorem 1, we shall see that the result concerns in fact only the Hodge structure on $H^{n}(X)_{\text {prim }}$ and not the effective geometry of $X$. In fact we get as well:

THEOREM 3. Let $X$ be a general hypersurface of degree $d \geq 2 n-2+3 g, g \geq 2$ or $d \geq 2 n+2, g=1$, in $\mathbb{P}^{n+1}$. Then there exists no non-zero morphism of Hodge structures

$$
H^{n}(X, \mathbb{Q})_{\text {prim }} \rightarrow H^{n}(Y, \mathbb{Q}),
$$

where $Y$ is rationally swept out by curves of genus $g$.

Combining this statement with the higher dimensional generalization of Mumford's theorem on 0-cycles on surfaces [17], this implies in particular that for $d \geq 2 n+2, X$ general, there exists no correspondence $\Gamma \in C H^{n}(Y \times X)$ inducing a surjective map

$$
\Gamma_{*}: C H_{0}(Y)_{0} \rightarrow C H_{0}(X)_{0},
$$

where $Y$ admits an elliptic fibration. Similarly, if $g \geq 2$ and $d \geq 2 n-2+3 g$, there exists no such correspondence $\Gamma \in C H^{n}(Y \times X)$ where $Y$ admits a fibration whose generic fiber is a genus $g$ curve. One may wonder whether these statements are true for any such hypersurface or only for the general one.

The paper is organized as follows: in Section 1, we recall briefly the proof of Nori's connectivity theorem for hypersurfaces in projective space, in order to prove a variant of it concerning families of hypersurfaces parameterized by subvarieties of the moduli space which are of small codimension. This will show us that for any family of hypersurfaces parameterized by a subvariety of the moduli space which is of small codimension, the Hodge level of the cohomology groups of the total space of the family is small.

The next section is devoted to the proof of Theorem 1.

In Section 3, we prove the applications of this result described above.

ACKNOWLEDGEMENTS. I would like to thank J. Harris who started me thinking to these problems and H. Clemens for very interesting discussions and comments.

This work has been essentially done at the University La Sapienza, and I would like to thank the organizers of the trimester "Moduli spaces, Lie theory, interactions with Physics" for the excellent working conditions and atmosphere I found there. 


\section{1. - Nori's connectivity theorem for hypersurfaces}

This section is devoted to an intermediate result (Theorem 4) toward the proof of Theorem 1. This result is a variant of Nori's connectivity theorem [18], or more precisely of its explicit version for hypersurfaces in projective space (cf [21], 8.1, [22]). In [1], [19], a sharper study of similar explicit bounds can be found.

Nori's theorem concerns the cohomology of locally complete families of sufficiently ample complete intersections in any smooth complex projective variety, parameterized by a smooth quasiprojective basis. The explicit version in case of hypersurfaces in projective space makes precise how ample the hypersurfaces must be.

Here, we consider a variant of this explicit version, where instead of locally complete families, we consider families of hypersurfaces whose moduli are of small codimension in the moduli space of all hypersurfaces.

We consider hypersurfaces of degree $d$ in $\mathbb{P}^{n+1}$, and we assume that $d \geq$ $n+2$. Fix an integer $r$ such that $1 \leq r \leq n$, and let $\gamma$ be the round-up of $\frac{r-\overline{1}}{2}$. Denote by $U \subset H^{0}\left(\mathcal{O}_{\mathbb{P} n+1}(d)\right)$ the open set parametrizing smooth hypersurfaces. Let $\rho: \mathcal{M} \rightarrow U$ be a morphism, where $\mathcal{M}$ is smooth quasi-projective. We assume that Corank $\rho$ is constant equal to $C$. We also assume for simplicity that $\operatorname{Im} \rho$ is stable under the action of $G l(n+2)$. Let $\mathcal{X}_{U}$ be the universal hypersurface parameterized by $U$ and

$$
\mathcal{X}_{\mathcal{M}}:=\mathcal{X}_{U} \times_{U} \mathcal{M}
$$

Let

$$
j: \mathcal{X}_{\mathcal{M}} \hookrightarrow \mathcal{M} \times \mathbb{P}^{n+1}
$$

be the natural embedding. $\mathcal{X}_{\mathcal{M}}$ is a smooth quasi-projective variety, hence its cohomology groups carry mixed Hodge structures with associated Hodge filtration $F^{i} H^{k}\left(\mathcal{X}_{\mathcal{M}}, \mathbb{C}\right)$.

THEOREM 4.

i) Assume that

$$
(d+1) r \geq 2 n+C+2 .
$$

Then, the restriction map

$$
j^{*}: F^{n} H^{2 n-r}\left(\mathcal{M} \times \mathbb{P}^{n+1}, \mathbb{C}\right) \rightarrow F^{n} H^{2 n-r}\left(\mathcal{X}_{\mathcal{M}}, \mathbb{C}\right)
$$

ii) If

is surjective.

$$
(\gamma+1) d \geq 2 n+1-r+C,
$$

then for any $i \geq 1$, the restriction map

$$
j^{*}: H^{2 n-r-i}\left(\mathcal{M} \times \mathbb{P}^{n+1}, \mathbb{C}\right) \rightarrow H^{2 n-r-i}\left(\mathcal{X}_{\mathcal{M}}, \mathbb{C}\right)
$$

is surjective. 
The proof mimics the proof given in [21], 8.1 (which is nothing but the original proof of Nori, in the case of hypersurfaces of projective space). For the reader who knows already the arguments given there, let us just say that the proof in [21], 8.1 reduces first to showing that complexes built from the infinitesimal variations of Hodge structures (IVHS) on the primitive part of the cohomology of hypersurfaces are exact in a certain range. Via Carlson-Griffiths descrition of these IVHS, using the Griffiths description of the Hodge structure of an hypersurface via residues, these complexes are pointwise identified to Koszul complexes of the Jacobian ring of the considered hypersurface. The exactness of these Koszul complexes then uses a theorem due to M. Green on the Koszul cohomology of projective space. Our variant is then obtained by applying Green's refined theorem, which concerns Koszul cohomology of projective space, with respect to a non-complete base point free linear system of small codimension.

We try now to go a little more into the details, without reproducing the whole proof in [21], 8.1. We refer to loc. cit. for complete proofs of the steps sketched below.

Proof of Theorem 4.

i) One first reduces the assertion, as in [18], to proving:

Under the assumption (1.2), the restriction map

$$
j^{*}: H^{l}\left(\Omega_{\mathcal{M} \times \mathbb{P}^{n+1}}^{k}\right) \rightarrow H^{l}\left(\Omega_{\mathcal{X}_{\mathcal{M}}}^{k}\right)
$$

is bijective, for $l \leq n-r, k+l \leq 2 n-r$.

This step uses the mixed Hodge structure on relative cohomology and the Frölicher spectral sequence: the relative cohomology of the pair $\left(\mathcal{M} \times \mathbb{P}^{n+1}, \mathcal{X}_{\mathcal{M}}\right)$ is equal to the hypercohomology of the complex

$$
\Omega_{\mathcal{M} \times \mathbb{P}^{n+1}, \mathcal{X}_{\mathcal{M}}}:=\operatorname{Ker}\left(j^{*}: \Omega_{\mathcal{M} \times \mathbb{P}^{n+1}} \rightarrow \Omega_{\mathcal{X}_{\mathcal{M}}}\right) .
$$

The Frölicher spectral sequence considered here is the spectral sequence associated to the naïve filtration on $\Omega_{\mathcal{M} \times \mathbb{P}^{n+1}, \mathcal{X}_{\mathcal{M}}}$.

Next denote respectively by $\pi_{X}, \pi_{\mathbb{P}}$ the natural maps

$$
\mathcal{X}_{\mathcal{M}} \rightarrow \mathcal{M}, \mathcal{M} \times \mathbb{P}^{n+1} \rightarrow \mathcal{M} .
$$

A Leray spectral sequence argument shows that it suffices to prove that under the assumption (1.2) one has:

(1.4) The restriction map $j^{*}: R^{l} \pi_{\mathbb{P} *}\left(\Omega_{\mathcal{M} \times \mathbb{P}}^{k} d+1\right) \rightarrow R^{l} \pi_{X *}\left(\Omega_{\mathcal{X}_{\mathcal{M}}}^{k}\right)$ is bijective for $l \leq n-r, k+l \leq 2 n-r$.

Let now $\mathcal{H}_{\text {prim }}^{n}, \mathcal{H}_{\text {prim }}^{p, q}, p+q=n$ be the Hodge bundles associated to the variation of Hodge structure on the primitive cohomology of the family 
$\pi_{X}: \mathcal{X}_{\mathcal{M}} \rightarrow \mathcal{M}$. The infinitesimal variation of Hodge structure on the primitive cohomology of the fibers of $\pi_{X}$ is described by maps

$$
\bar{\nabla}: \mathcal{H}_{\text {prim }}^{p, q} \rightarrow \mathcal{H}_{\text {prim }}^{p-1, q+1} \otimes \Omega_{\mathcal{M}}
$$

and they can be extended in the obvious way to produce a complex:

$$
\ldots \mathcal{H}_{\text {prim }}^{p+1, q-1} \otimes \Omega_{\mathcal{M}}^{s-1} \stackrel{\bar{\nabla}}{\rightarrow} \mathcal{H}_{\text {prim }}^{p, q} \otimes \Omega_{\mathcal{M}}^{s} \stackrel{\bar{\nabla}}{\rightarrow} \mathcal{H}_{\text {prim }}^{p-1, q+1} \otimes \Omega_{\mathcal{M}}^{s+1} \ldots
$$

One shows, using the filtration of $\Omega_{\mathcal{X}}^{k}$ by the subbundles $\pi_{X}^{*} \Omega_{B}^{s} \wedge \Omega_{\mathcal{X}}^{k-s}$ and the associated spectral sequence ( $\operatorname{cf}[21], 5.2 .1)$, that (1.4) is equivalent to the following

The sequence (1.6) is exact at the middle for $q \leq n-r, p+s+q \leq 2 n-r$.

Note that since $p+q=n$, the last inequality reduces to $s \leq n-r$.

It is convenient to dualize (1.6) using Serre duality, which gives:

$$
\mathcal{H}_{\text {prim }}^{q+1, p-1} \otimes \bigwedge^{s+1} T_{\mathcal{M}} \stackrel{t \bar{\nabla}}{\rightarrow} \mathcal{H}_{\text {prim }}^{q, p} \otimes \bigwedge^{s} T_{\mathcal{M}}^{s} \stackrel{t \bar{\nabla}}{\rightarrow} \mathcal{H}_{\text {prim }}^{q-1, p+1} \otimes \bigwedge^{s-1} T_{\mathcal{M}}^{s+1}
$$

We finally use Griffiths, Griffiths-Carlson description of the IVHS of hypersurfaces ([15], [2]) to describe the complex (1.7) at the point $f \in \mathcal{M}$ as follows. We have the map $\rho_{*}: T_{\mathcal{M}, f} \rightarrow T_{U, f}=S^{d}$, where $S$ is the polynomial ring in $n+2$ variables. Next the residue map provides isomorphisms

$$
R_{f}^{-n-2+d(p+1)} \cong H_{\text {prim }}^{q, p}\left(X_{f}\right),
$$

where $R_{f}:=S / J_{f}$ is the Jacobian ideal of $f$, and $R_{f}^{k}$ denotes its degree $k$ component. The map $\bar{\nabla}$ in (1.5) identifies then, up to a coefficient, to the map given by $\rho_{*}$ and multiplication:

$$
R_{f}^{-n-2+d(p+1)} \rightarrow \operatorname{Hom}\left(T_{\mathcal{M}, f}, R_{f}^{-n-2+d(p+2)}\right) .
$$

It follows from this that the sequence (1.7) identifies to the following piece of the Koszul complex of the Jacobian ring $R_{f}$ with respect to the action of $T_{\mathcal{M}, f}$ on it by multiplication:

$$
\begin{aligned}
R_{f}^{-n-2+d p} & \otimes \bigwedge^{s+1} T_{\mathcal{M}, f} \stackrel{\delta}{\rightarrow} R_{f}^{-n-2+d(p+1)} \otimes \bigwedge^{s} T_{\mathcal{M}, f} \\
& \stackrel{\delta}{\rightarrow} R_{f}^{-n-2+d(p+2)} \otimes \bigwedge^{s-1} T_{\mathcal{M}, f} .
\end{aligned}
$$

Now, by assumption, if $W$ is the image of $\rho_{*}, W \subset S^{d}$ is a base-point free linear system, because it contains the jacobian ideal $J_{f}^{d}$, and it satisfies codim $W=C$.

One verifies that it suffices to check exactness at the middle of the exact sequences (1.8) in the considered range, with $T_{\mathcal{M}, f}$ replaced with $W$. This last fact is then a consequence of the following theorem due to $\mathrm{M}$. Green: 
THEOREM 5 [14]. Let $W \subset S^{d}$ be a base-point free linear system. Then the following sequence, where the differentials are the Koszul differentials

$$
S^{-n-2+d p} \otimes \bigwedge^{s+1} W \stackrel{\delta}{\rightarrow} S^{-n-2+d(p+1)} \otimes \bigwedge^{s} W \stackrel{\delta}{\rightarrow} S^{-n-2+d(p+2)} \otimes \bigwedge^{s-1} W
$$

is exact for $-n-2+d p \geq s+\operatorname{codim} W$.

Using the fact that the Jacobian ideal is generated by a regular sequence in degree $d-1$, which provides a very simple resolution of $R_{f}$ by graded free $S$-modules, one then shows that the same is true when $S^{i}$ is replaced with $R_{f}^{i}$ in (1.9), at least if $-n-2+d(p+1) \geq d-1$.

We now conclude the proof of i). We just proved that (1.6) is exact at the middle if

$$
-n-2+d p \geq s+C,-n-2+d(p+1) \geq d-1 .
$$

Since we assumed $d \geq n+2$, the second inequality is satisfied when $p \geq 1$. Next, if $q \leq n-r, s \leq n-r$, we have

$$
p \geq r \geq 1, s \leq n-r .
$$

Hence, the exactness of (1.6) in the range $q \leq n-r, s \leq n-r$ will follow from the inequality

$$
-n-2+d r \geq n-r+C,
$$

that is (1.2).

ii) The proof is entirely similar, and we just sketch it in order to see where the numerical assumption is used. Following [18], we first observe that, in order to get the surjectivity of the restriction map:

$$
j^{*}: H^{2 n-r-i}\left(\mathcal{M} \times \mathbb{P}^{n+1}, \mathbb{C}\right) \rightarrow H^{2 n-r-i}\left(\mathcal{X}_{\mathcal{M}}, \mathbb{C}\right),
$$

it suffices to show the surjectivity of the restriction map:

$$
j^{*}: F^{n-r+\gamma_{i}} H^{2 n-r-i}\left(\mathcal{M} \times \mathbb{P}^{n+1}, \mathbb{C}\right) \rightarrow F^{n-r+\gamma_{i}} H^{2 n-r-i}\left(\mathcal{X}_{\mathcal{M}}, \mathbb{C}\right),
$$

where $\gamma_{i}$ is the round-up of $\frac{r-i}{2}$. Indeed, let $K$ be the cokernel

$$
H^{2 n-r-i}\left(\mathcal{X}_{\mathcal{M}}, \mathbb{Q}\right) / j^{*} H^{2 n-r-i}\left(\mathcal{M} \times \mathbb{P}^{n+1}, \mathbb{Q}\right) .
$$

It has a quotient mixed Hodge structure whose weights are $\geq 2 n-r-i$, because $\mathcal{X}_{\mathcal{M}}$ is smooth (cf [10]). The surjectivity of

$$
j^{*}: F^{n-r+\gamma_{i}} H^{2 n-r-i}\left(\mathcal{M} \times \mathbb{P}^{n+1}, \mathbb{C}\right) \rightarrow F^{n-r+\gamma_{i}} H^{2 n-r-i}\left(\mathcal{X}_{\mathcal{M}}, \mathbb{C}\right),
$$


then says that $F^{n-r+\gamma_{i}} K_{\mathbb{C}}=0$. But the round-up of $\frac{2 n-r-i}{2}$ is $n-r+\gamma_{i}$. Applying Hodge symmetry to the weight graded pieces of $K^{2}$ (which carry pure Hodge structures of weight $\geq 2 n-r-i$ ), we see that

$$
F^{n-r+\gamma_{i}} K_{\mathbb{C}}=0 \Rightarrow K=0 .
$$

As in the previous proof, we reduce now the proof of the surjectivity of (1.10) to showing:

(1.11) The restriction map $j^{*}: R^{l} \pi_{\mathbb{P}_{*}}\left(\Omega_{\mathcal{M} \times \mathbb{P}^{n+1}}^{k}\right) \rightarrow R^{l} \pi_{X *}\left(\Omega_{\mathcal{X}_{\mathcal{M}}}^{k}\right)$ is bijective for $l \leq n-i-\gamma_{i}, k+l \leq 2 n-r-i$.

Expressing as in the previous proof the cohomology groups above with the help of the IVHS on the primitive cohomology of the fibers of $\pi_{X}$, this is reduced to proving:

The sequence (1.6) is exact at the middle for $q \leq n-i-\gamma_{i}, p+q=n, p+s+q \leq$ $2 n-r-i$.

Using the Carlson-Griffiths theory, we are now reduced to prove:

The following sequence:

$$
\begin{aligned}
R_{f}^{-n-2+d p} \otimes \bigwedge^{s+1} T_{\mathcal{M}, f} & \stackrel{\delta}{\rightarrow} R_{f}^{-n-2+d(p+1)} \otimes \bigwedge^{s} T_{\mathcal{M}, f} \\
& \stackrel{\delta}{\rightarrow} R_{f}^{-n-2+d(p+2)} \otimes \bigwedge^{s-1} T_{\mathcal{M}, f}
\end{aligned}
$$

is exact for $p \geq \gamma_{i}+i, s \leq n-r-i$.

As in the previous proof, we now apply Theorem 5 and conclude that the last statement is true if

$$
-n-2+d\left(\gamma_{i}+i\right) \geq C+n-r-i .
$$

Now it is clear that the $\gamma_{i}+i$ are increasing with $i$, while the $C+n-r-i$ are decreasing with $i$. Hence it suffices to have (1.13) satisfied for $i=1$, which is exactly inequality (1.13).

Denoting by $H^{2 n-r}\left(\mathcal{X}_{\mathcal{M}}\right)_{\text {prim }}$ the quotient

$$
H^{2 n-r}\left(\mathcal{X}_{\mathcal{M}}\right) / j^{*}\left(H^{2 n-r}\left(\mathcal{M} \times \mathbb{P}^{n+1}\right)\right),
$$

we shall only be interested with the pure part

$$
W_{2 n-r} H^{2 n-r}\left(\mathcal{X}_{\mathcal{M}}\right)_{\text {prim }},
$$

which is the part of the cohomology which comes from any smooth projective compactification of $\mathcal{X}_{\mathcal{M}}$ [10]. It carries a pure Hodge structure of weight $2 n-r$. 
COROLLARY 1. Under the assumptions of Theorem 4, the Hodge structure on $W_{2 n-r} H^{2 n-r}\left(\mathcal{X}_{\mathcal{M}}\right)_{\text {prim }}$ is of Hodge level $\leq r-2$.

PROOF. Recall that the Hodge level of a pure Hodge structure $H, H_{\mathbb{C}}=$ $\oplus H^{p, q}$ is

$$
\operatorname{Max}\left\{p-q, H^{p, q} \neq 0\right\} .
$$

Since we know that $F^{n} W_{2 n-r} H^{2 n-r}\left(\mathcal{X}_{\mathcal{M}}\right)_{\text {prim }}=0$, we have

$$
H^{p, q}\left(W_{2 n-r} H^{2 n-r}\left(\mathcal{X}_{\mathcal{M}}\right)_{\text {prim }}\right)=0, \text { for } p \geq n .
$$

Since $H^{p, q}=0$ for $p+q \neq 2 n-r$, it follows that the Hodge level is $\leq n-1-(2 n-r-(n-1))=r-2$.

\section{2. - Proof of Theorem 1}

We prove Theorem 1 by contradiction. So assume the assumptions $(0.1)$ are satisfied, but that the general $X$ of degree $d$ in $\mathbb{P}^{n+1}$ is rationally swept-out by varieties from the family $\mathcal{Y}$.

Using Chow varieties, or relative Hilbert schemes, we see that there exist countably many quasi-projective varieties $\mathcal{B}_{k}$ parameterizing triples $\left(t, f, \phi_{s}\right)$, where $t \in \mathcal{S}, f \in U$, and $\phi_{s}$ is a rational map $\phi_{s}: Y_{s} \rightarrow X_{f}$ which is generically finite onto its image. Indeed, such a $\phi_{s}$ can be identified to its graph, which is a $r$-dimensional subvariety of the product $Y_{s} \times X_{f}$, which has to be of degree 1 over the first factor.

For fixed generic $f$, our assumption implies that the images of such $\phi_{s}$ fill-in $X_{f}$.

For each $k$, let

$$
\pi_{k}: \mathcal{K}_{k} \rightarrow \mathcal{B}_{k}
$$

be the family which is pulled-back, via the first projection $\mathcal{B}_{k} \rightarrow \mathcal{S}$, from the family $\mathcal{Y} \rightarrow \mathcal{S}$. By definition of $\mathcal{B}_{k}$ as a Hilbert scheme, there is the universal rational map

$$
\Phi_{k}: \mathcal{K}_{k} \rightarrow \mathcal{X}_{U}
$$

where, as in the previous section, $\mathcal{X}_{U}$ is the universal hypersurface parameterized by $U$. Note that $\Phi_{k}$ makes the following diagram commutative:

$$
\begin{aligned}
& \Phi_{k}: \mathcal{K}_{k} \rightarrow \mathcal{X}_{U} \\
& \downarrow \pi_{k} \quad \downarrow \\
& p r_{2}: \mathcal{B}_{k} \rightarrow U .
\end{aligned}
$$

Our assumption implies that the union of the images of $\Phi_{k}$ contains a subset of $\mathcal{X}_{U}$ which is the complementary set of a countable union of proper closed algebraic subsets. By Baire, it follows that for some $k_{0}, \Phi_{k_{0}}$ is dominating. 
In the sequel, we shall use the notations $\pi, \mathcal{B}, \mathcal{K}, \Phi$, for $\pi_{k_{0}}, \mathcal{B}_{k_{0}}, \mathcal{K}_{k_{0}}, \Phi_{k_{0}}$ respectively.

We shall denote by $B_{f}$ the (generic) fiber of the second projection $\mathcal{B} \rightarrow U$ and $\pi_{f}: K_{f} \rightarrow B_{f}$ the induced family. By taking desingularizations, we may assume that $\mathcal{B}$ is smooth, and since by assumption the map $\pi$ is smooth, $\mathcal{K}$ is smooth too. Since $f$ is generic, $B_{f}$ and $K_{f}$ are then also smooth. Finally, we may, up to replacing $\mathcal{B}$ by a closed subvariety, assume that the restriction $\Phi_{f}: K_{f} \rightarrow X_{f}$ of $\Phi$ to $K_{f}$ is generically finite and dominating. (This is because the restriction of $\Phi$ to the generic fibers of $\pi$ is generically finite.) In particular, $\operatorname{dim} B_{f}=n-r$.

We fix now a generic point $t \in \operatorname{Im} p r_{1} \subset \mathcal{S}$. We let

$$
\mathcal{B}_{t}:=p r_{1}^{-1}(t), \mathcal{K}_{t}:=\pi^{-1}\left(\mathcal{B}_{t}\right) .
$$

We observe that $\mathcal{K}_{t}$ is naturally isomorphic to $Y_{t} \times \mathcal{B}_{t}$. Furthermore, since

$$
\operatorname{dim} I m p r_{1} \leq \operatorname{dim} \mathcal{S} \leq C,
$$

we have $\operatorname{codim}\left(\mathcal{B}_{t} \subset \mathcal{B}\right) \leq C$.

The second projection $p r_{2}: \mathcal{B} \rightarrow U$ is dominating, so can be assumed to be submersive, after shrinking $\mathcal{B}$. It follows that the corank of $p r_{2 \mid \mathcal{B}_{t}}$ is $\leq C$, and after shrinking $\mathcal{B}_{t}$, we may assume it is constant and $\leq C$ on $\mathcal{B}_{t}$.

Let $\mathcal{X}_{\mathcal{B}_{t}}:=\mathcal{X}_{U} \times_{U} \mathcal{B}_{t}$. The map $\Phi: \mathcal{K} \rightarrow \mathcal{X}_{U}$ lifts to a map

$$
\widetilde{\Phi}: \mathcal{K} \rightarrow \mathcal{X}_{\mathcal{B}},
$$

because of the commutativity of the diagram (2.14). $\widetilde{\Phi}$ takes the value $\phi_{s}$ at the point $\left(t, f, \phi_{s}\right)$ of $\mathcal{B}$.

This map restricts in turn to a map

$$
\widetilde{\Phi}_{t}: \mathcal{K}_{t} \cong Y_{t} \times \mathcal{B}_{t} \rightarrow \mathcal{X}_{\mathcal{B}_{t}},
$$

which is over $\mathcal{B}_{t}$.

The relative graph of $\widetilde{\Phi}_{t}$ provides then a codimension $n$ subvariety in the fibred product

$$
\mathcal{K}_{t} \times \mathcal{B}_{t} \mathcal{X}_{\mathcal{B}_{t}} \cong Y_{t} \times \mathcal{X}_{\mathcal{B}_{t}}
$$

The proof concludes now as follows: we know that the map $\mathcal{B}_{t} \rightarrow U$ is of constant corank $\leq C$, and by assumption, the inequality (1.2) needed in Corollary 1 is satisfied, so Corollary 1 holds, that is, the Hodge structure on $W_{2 n-r} H^{2 n-r}\left(\mathcal{X}_{\mathcal{B}_{t}}, \mathbb{Q}\right)_{\text {prim }}$ has Hodge level $<r$. This contradicts the following Proposition 2.

Proposition 2. The point $t \in \mathcal{S}$ being generic as above, let

$$
\gamma_{t} \in H^{2 n}\left(Y_{t} \times \mathcal{X}_{\mathcal{B}_{t}}, \mathbb{Q}\right)
$$

be the rational cohomology class of the graph of $\widetilde{\Phi}_{t}$. Then $\gamma_{t}$ induces a morphism of mixed Hodge structures

$$
\gamma_{t *}: H^{r}\left(Y_{t}, \mathbb{Q}\right) \rightarrow H^{2 n-r}\left(\mathcal{X}_{\mathcal{B}_{t}}, \mathbb{Q}\right)_{\text {prim }}
$$

whose image has Hodge level $r$. 
Remark 3. The mixed Hodge structure on $H^{r}\left(Y_{t}, \mathbb{Q}\right)$ is pure as is the mixed Hodge structure on $\operatorname{Im} \gamma_{t *}$. Hence it makes sense to speak of the Hodge level of the image.

Remark 4. The fact that $\operatorname{Im} \gamma_{t *}$ has Hodge level $r$ is equivalent to the fact that $\gamma_{t *}$ does not vanish identically on $H^{r, 0}\left(Y_{t}\right)$, or more generally on the subspace $H^{r}\left(Y_{t}, \mathbb{Q}\right)_{t r}$ introduced later on in the proof.

Proof of Proposition 2. The graph of $\widetilde{\Phi}$ lies in $\mathcal{K} \times_{\mathcal{B}} \mathcal{X}_{\mathcal{B}}$ and is of codimension $n$ there. Let $\gamma \in H^{2 n}\left(\mathcal{K} \times_{\mathcal{B}} \mathcal{X}_{\mathcal{B}}, \mathbb{Q}\right)$ be its rational cohomology class. Of course

$$
\gamma_{t}=\gamma_{\mid \mathcal{X}_{\mathcal{B}_{t}}}
$$

Let $\tilde{\pi}: \mathcal{K} \times_{\mathcal{B}} \mathcal{X}_{\mathcal{B}} \rightarrow \mathcal{X}_{\mathcal{B}}$ be the second projection. Our first task is basically to replace $\gamma$ by a class $\gamma^{\prime}$ which lies in the $r$-th Leray level of $H^{2 n}\left(\mathcal{K} \times_{\mathcal{B}} \mathcal{X}_{\mathcal{B}}, \mathbb{Q}\right)$ with respect to $\tilde{\pi}$ : Let $\mathcal{L}$ be a relatively ample line bundle on $\mathcal{Y} \rightarrow \mathcal{S}$, and let $\widetilde{\mathcal{L}}$ be its pull-back to $\mathcal{K} \times_{\mathcal{B}} \mathcal{X}_{\mathcal{B}}$ via the natural composite map

$$
\mathcal{K} \times_{\mathcal{B}} \mathcal{X}_{\mathcal{B}} \rightarrow \mathcal{K} \rightarrow \mathcal{Y} .
$$

Then $\widetilde{\mathcal{L}}$ is relatively ample w.r.t. $\tilde{\pi}$.

The hard Lefschetz theorem provides isomorphisms

$$
c_{1}(\mathcal{L})^{i} \cup: R^{r-i} \tilde{\pi}_{*} \mathbb{Q} \cong R^{r+i} \tilde{\pi}_{*} \mathbb{Q}, i \geq 0,
$$

and correspondingly isomorphisms:

$$
c_{1}(\mathcal{L})^{i} \cup: H^{2 n-r-i}\left(\mathcal{X}_{\mathcal{B}}, R^{r-i} \tilde{\pi}_{*} \mathbb{Q}\right) \cong H^{2 n-r-i}\left(\mathcal{X}_{\mathcal{B}}, R^{r+i} \tilde{\pi}_{*} \mathbb{Q}\right), i \geq 0 .
$$

These isomorphisms are isomorphisms of mixed Hodge structures. Furthermore, by Deligne's theorem [9], the Leray spectral sequence of $\tilde{\pi}$ degenerates at $E_{2}$, so that the above groups are graded pieces of

$$
H^{2 n-2 i}\left(\mathcal{K} \times_{\mathcal{B}} \mathcal{X}_{\mathcal{B}}, \mathbb{Q}\right), H^{2 n}\left(\mathcal{K} \times_{\mathcal{B}} \mathcal{X}_{\mathcal{B}}, \mathbb{Q}\right)
$$

respectively. It follows that we can write

$$
\gamma=\gamma^{\prime}+\sum_{r \geq i>0} c_{1}(\widetilde{\mathcal{L}})^{i} \cup \gamma_{i},
$$

where $\gamma^{\prime} \in H^{2 n}\left(\mathcal{K} \times_{\mathcal{B}} \mathcal{X}_{\mathcal{B}}, \mathbb{Q}\right)$ lies in the $r$-th level of the Leray filtration relative to $\tilde{\pi}$, and $\gamma_{i} \in H^{2 n-2 i}\left(\mathcal{K} \times_{\mathcal{B}} \mathcal{X}_{\mathcal{B}}, \mathbb{Q}\right)$. By strictness of the morphisms of mixed Hodge structures with respect to both filtrations, we may even assume that $\gamma^{\prime}$ and $\gamma_{i}$ are Hodge classes, that is lie respectively in

$$
\begin{gathered}
W_{2 n} H^{2 n}\left(\mathcal{K} \times_{\mathcal{B}} \mathcal{X}_{\mathcal{B}}, \mathbb{Q}\right) \cap F^{n} H^{2 n}\left(\mathcal{K} \times_{\mathcal{B}} \mathcal{X}_{\mathcal{B}}, \mathbb{Q}\right), \\
W_{2 n-2 i} H^{2 n-2 i}\left(\mathcal{K} \times_{\mathcal{B}} \mathcal{X}_{\mathcal{B}}, \mathbb{Q}\right) \cap F^{n-i} H^{2 n-2 i}\left(\mathcal{K} \times_{\mathcal{B}} \mathcal{X}_{\mathcal{B}}, \mathbb{Q}\right) .
\end{gathered}
$$


Restrict now everything to

$$
\mathcal{K}_{t} \times_{\mathcal{B}_{t}} \mathcal{X}_{\mathcal{B}_{t}} \cong Y_{t} \times \mathcal{X}_{\mathcal{B}_{t}} .
$$

Since $\widetilde{\mathcal{L}}$ is the pull-back of a line bundle on $\mathcal{Y}$, its restriction $\widetilde{\mathcal{L}}_{t}$ to $\mathcal{K}_{t} \times_{\mathcal{B}_{t}} \mathcal{X}_{\mathcal{B}_{t}}$ is of the form $p r_{1}^{*} L$, for some line bundle $L$ on $Y_{t}$, where $p r_{1}$ is defined using the isomorphism (2.17). It follows that each

$$
\gamma_{i, t}:=c_{1}(\widetilde{\mathcal{L}})^{i} \cup \gamma_{i \mid \mathcal{K}_{t} \times \mathcal{B}_{t}} \mathcal{X}_{\mathcal{B}_{t}}
$$

induces a morphism of mixed Hodge structures

$$
H^{r}\left(Y_{t}, \mathbb{Q}\right) \rightarrow H^{2 n-r}\left(\mathcal{X}_{\mathcal{B}_{t}}, \mathbb{Q}\right),
$$

which factors through the map $c_{1}(L)^{i} \cup: H^{r}\left(Y_{t}, \mathbb{Q}\right) \rightarrow H^{r+2 i}\left(Y_{t}, \mathbb{Q}\right)$. So, since $\operatorname{dim} Y_{t}=r$, each such morphism has an image which is of Hodge level $<r$ for $i>0$. It follows that it suffices to prove the conclusion of Proposition 2 for $\gamma^{\prime}$.

We observe now the following: $\gamma^{\prime}$ lies in the $r$-th level of the Leray filtration of $H^{2 n}\left(\mathcal{K} \times_{\mathcal{B}} \mathcal{X}_{\mathcal{B}}, \mathbb{Q}\right)$ relative to $\tilde{\pi}$, hence, using the degeneracy at $E_{2}$ of this Leray spectral sequence, it projects to an element $\gamma^{\prime \prime}$ in

$$
H^{2 n-r}\left(\mathcal{X}_{\mathcal{B}}, R^{r} \tilde{\pi}_{*} \mathbb{Q}\right),
$$

which is the $E_{2}^{r, n-r}=E_{\infty}^{r, n-r}$ term of the Leray spectral sequence of $\tilde{\pi}$.

Denote by $\psi$ the composite map

$$
\mathcal{X}_{\mathcal{B}} \rightarrow \mathcal{B} \rightarrow \mathcal{S} .
$$

Observe that the family

$$
\tilde{\pi}: \mathcal{K} \times_{\mathcal{B}} \mathcal{X}_{\mathcal{B}} \rightarrow \mathcal{X}_{\mathcal{B}}
$$

is pulled-back via $\psi$ from the family $\mathcal{Y} \rightarrow \mathcal{S}$. It follows that the local system is also pulled-back:

$$
R^{r} \tilde{\pi}_{*} \mathbb{Q}=\psi^{-1} H^{r},
$$

for some local system $H^{r}$ of $\mathbb{Q}$-vector spaces on $\mathcal{S}$. Hence the $E_{2}$ terms of the Leray spectral sequence relative to $\psi$ and to the sheaf $R^{r} \tilde{\pi}_{*} \mathbb{Q}$ on $\mathcal{X}_{\mathcal{B}}$ are

$$
H^{i}\left(\mathcal{S}, R^{2 n-r-i} \psi_{*} \mathbb{Q} \otimes_{\mathbb{Q}} H^{r}\right) .
$$

From now on we assume that the map $p r_{1}: \mathcal{B} \rightarrow \mathcal{S}$ is surjective, and topologically locally trivial, which we can do, replacing $\mathcal{S}$ by a Zariski open set in Im $\mathrm{pr}_{1}$. The local system $H^{r}$ contains a sub-local system which via Poincaré duality is also canonically a direct summand, whose fiber at the general point of $\mathcal{S}$ is the maximal sub-Hodge structure of $H^{r}\left(Y_{t}, \mathbb{Q}\right)$ which is not of maximal Hodge level. Let us denote this sub-local system by $H_{<r}^{r}$, and the quotient 
by $H_{t r}^{r}$. $H_{t r}^{r}$ can also be defined as the minimal sub-variation of Hodge structure of $H^{r}$ whose corresponding $(r, 0)$-part contains the whole bundle $\mathcal{H}^{r, 0}$ with fiber $H^{r, 0}\left(Y_{t}\right)$ at $t \in \mathcal{S}$. So we have an orthogonal decomposition:

$$
H^{r}=H_{<r}^{r} \oplus H_{t r}^{r} .
$$

Let $R^{r} \tilde{\pi}_{*} \mathbb{Q}_{t r}:=\psi^{-1} H_{t r}^{r}$. With these notations, we have the component $\gamma_{t r}^{\prime \prime}$ of $\gamma^{\prime \prime}$, which lies in

$$
H^{2 n-r}\left(\mathcal{X}_{\mathcal{B}}, R^{r} \tilde{\pi}_{*} \mathbb{Q}_{t r}\right),
$$

and as above, the spectral sequence abutting to this last group has $E_{2}$-term

$$
H^{i}\left(\mathcal{S}, R^{2 n-r-i} \psi_{*} \mathbb{Q} \otimes_{\mathbb{Q}} H_{t r}^{r}\right) .
$$

We apply now Theorem 4, ii). The fibers of the maps $\psi$ are the $\mathcal{X}_{\mathcal{B}_{t}}$ hence they satisfy the vanishing

$$
H^{2 n-r-i}\left(\mathcal{X}_{\mathcal{B}_{t}}, \mathbb{Q}\right)_{\text {prim }}=0, i>0 .
$$

It follows that for $i>0$, we have

$$
H^{i}\left(\mathcal{S}, R^{2 n-r-i} \psi_{*} \mathbb{Q}_{\text {prim }} \otimes_{\mathbb{Q}} H_{t r}^{r}\right)=0 .
$$

Hence we conclude that up to classes coming from

$$
H^{2 n-r}\left(\mathbb{P}^{n+1} \times \mathcal{B}, R^{r}(I d \times \pi)_{*} \mathbb{Q}_{t r}\right),
$$

the class $\gamma_{t r}^{\prime \prime}$ is determined by its 0 -th Leray component, namely

$$
\gamma^{\prime \prime \prime} \in H^{0}\left(\mathcal{S}, R^{2 n-r} \psi_{*} \mathbb{Q}_{\text {prim }} \otimes_{\mathbb{Q}} H_{t r}^{r}\right) .
$$

Note that the value of $\gamma^{\prime \prime \prime}$ at the general point $t \in \mathcal{S}$ is, (using Poincare duality on $Y_{t}$, ) nothing but the restriction of the map $\gamma_{t *}^{\prime}$ or $\gamma_{t *}$ of (2.15) to the transcendental part $H^{r}\left(Y_{t}, \mathbb{Q}\right)_{t r}$.

Let us now conclude the proof by contradiction: assume the $\gamma_{t *}^{\prime}$ has an image which is of Hodge level $<r$ for general $t$. Then equivalently, its restriction to $H^{r}\left(Y_{t}, \mathbb{Q}\right)_{t r}$ vanishes. This means that $\gamma^{\prime \prime \prime}=0$, and by the reasoning above, this implies that $\gamma_{t r}^{\prime \prime}=0$ modulo classes coming from $H^{2 n-r}\left(\mathbb{P}^{n+1} \times \mathcal{B}, R^{r}(I d \times \pi)_{*} \mathbb{Q}_{t r}\right)$.

We restrict now everything to the fibers of

$$
\mathcal{X}_{\mathcal{B}} \rightarrow \mathcal{B} \rightarrow U, \quad \mathcal{K} \rightarrow \mathcal{B} \rightarrow U
$$

over $f \in U$. The fiber of $\mathcal{X}_{\mathcal{B}}$ over $f$ is $B_{f} \times X_{f}$, the fiber of $\mathcal{K}$ over $f$ is $K_{f}$, and correspondingly the fiber of $\mathcal{K} \times_{\mathcal{B}} \mathcal{X}_{\mathcal{B}}$ over $f$ is $K_{f} \times X_{f}$. The restriction of $\widetilde{\Phi}$ to $K_{f}$ is the map $\left(\pi_{f}, \Phi_{f}\right): K_{f} \rightarrow B_{f} \times X_{f}$ and so the graph of $\widetilde{\Phi}$ in 
$\mathcal{K} \times_{\mathcal{B}} \mathcal{X}_{\mathcal{B}}$ restricts to the graph of $\Phi_{f}$ in $K_{f} \times X_{f}$. The class $\gamma$ restricts to the class $\gamma_{f}$ of this graph.

Denote by $\gamma_{f}^{\prime}, \gamma_{f}^{\prime \prime}, \gamma_{f, t r}^{\prime \prime}$ the restrictions of the classes $\gamma^{\prime}, \gamma^{\prime \prime}, \gamma_{t r}^{\prime \prime}$ respectively to $K_{f} \times X_{f}$. The vanishing $\gamma_{t r}^{\prime \prime}=0$ implies the vanishing $\gamma_{f, t r}^{\prime \prime}=0$. Retracing through the definitions, the class

$$
\gamma_{f} \in H^{n}\left(K_{f}, \mathbb{Q}\right) \otimes H^{n}\left(X_{f}, \mathbb{Q}\right)_{\text {prim }},
$$

where the subscript "prim" here means "the orthogonal part to $H^{*}\left(\mathbb{P}^{n+1}, \mathbb{Q}\right)_{\mid X_{f}}$ ", decomposes as

$$
\gamma_{f}=\sum_{i>0} l^{i} \cup \gamma_{i, f}+\gamma_{f}^{\prime}
$$

where $l$ is the first Chern class of the line bundle on $K_{f}$ induced by $\mathcal{L}$ via the map $K_{f} \rightarrow \mathcal{Y}$. Here the $\gamma_{i, f}, \gamma^{\prime}$ are the restriction to $K_{f} \times X_{f}$ of the classes $\gamma_{i}, \gamma^{\prime}$ of (2.16). The class $\gamma_{f}^{\prime}$ belongs to $L_{r} H^{n}\left(K_{f}, \mathbb{Q}\right) \otimes H^{n}\left(X_{f}, \mathbb{Q}\right)_{\text {prim }}$, where $L_{r} H^{n}\left(K_{f}, \mathbb{Q}\right)$ is the $r$-th Leray level of $H^{n}\left(K_{f}, \mathbb{Q}\right)$ with respect to the Leray filtration relative to the map $\pi_{f}: K_{f} \rightarrow B_{f}$, and our conclusion is that its projection in

$$
H^{n-r}\left(B_{f}, R^{r} \pi_{f_{*}} \mathbb{Q}\right) \otimes H^{n}\left(X_{f}, \mathbb{Q}\right)_{\text {prim }}
$$

lies in $H^{n-r}\left(B_{f}, R^{r} \pi_{f_{*}} \mathbb{Q}_{<r}\right) \otimes H^{n}\left(X_{f}, \mathbb{Q}\right)_{\text {prim. }}$.

The contradiction now comes from the fact that since $\Phi_{f}$ is a dominating rational map, and the Hodge structure on $H^{n}\left(X_{f}, \mathbb{Q}\right)_{\text {prim }}$ has Hodge level $n$, (because $d \geq n+2$,) the associated map $\gamma_{f}^{*}$ has an image which is of Hodge level $n$.

On the other hand, the maps of the form $l^{i} \cup \gamma_{i, f}^{*}$ have images of Hodge level $<n$, as do the maps $\gamma_{i, f}^{*}$. Next, the only Leray piece of $H^{n}\left(K_{f}, \mathbb{Q}\right)$ whose weight $n$ part is of Hodge level $n$ is $H^{n-r}\left(R^{r} \pi_{f_{*}} \mathbb{Q}\right.$ ), (because $\operatorname{dim} B_{f}=n-r$,) and inside $H^{n-r}\left(R^{r} \pi_{f_{*}} \mathbb{Q}\right)$, the subspace $H^{n-r}\left(R^{r} \pi_{f_{*}} \mathbb{Q}<r\right)$ has Hodge level $<n$.

\section{3. - Rational maps from abelian varieties to Calabi-Yau hypersurfaces and other applications}

Proof of THEOREM 2. We want to show that for $r \geq 2$, the general Calabi-Yau hypersurfaces, that is hypersurfaces of degree $d=n+2$ in $\mathbb{P}^{n+1}$ are not rationally swept-out by $r$-dimensional abelian varieties. We apply Theorem 1: the family $\mathcal{Y} \rightarrow \mathcal{S}$ we consider is any locally universal family of abelian varieties. The moduli space of $r$-dimensional abelian varieties with given polarization type is of dimension $\frac{r(r+1)}{2}$. Hence the inequalities needed in Theorem 1 
become in this case:

$$
\begin{aligned}
(n+3) r & \geq 2 n+\frac{r(r+1)}{2}+2, \\
(\gamma+1)(n+2) & \geq 2 n-r+1+\frac{r(r+1)}{2} .
\end{aligned}
$$

It is not hard to check that this is satisfied for $2 \leq r \leq n$.

When $r=1$, inequality (0.1) is never satisfied so that our argument definitely does not apply to the study of elliptic curves in Calabi-Yau hypersurfaces. In fact we could adapt our proof of Theorem 2 to work as well for Calabi-Yau hypersurfaces in a product of projective spaces. On the other hand, certain generic Calabi-Yau hypersurfaces in a product of projective spaces are swept out by elliptic curves, eg the hypersurface of bidegree $(3,3)$ in $\mathbb{P}^{2} \times \mathbb{P}^{2}$. This shows that for $r=1$, a different argument has to be found. Note however that Theorem 2 has the following corollary, which says that Lang's and Clemens' conjectures contradict:

COROLLARY 2. If Lang's conjecture is true, any Calabi-Yau hypersurface $X$ of dimension $\geq 2$ has a divisor which is uniruled.

Proof. Indeed, if Lang's conjecture is true, then by Theorem 2, the general $X$ is rationally swept out by elliptic curves. One then applies the following result, which is proved in the three dimensional case in [7], lecture 22 (the proof however applies to any dimension $\geq 2$ ):

PROPOSITION 3. If a general Calabi-Yau hypersurface is rationally swept-out by elliptic curves, then it contains a divisor which is rationally swept-out by rational curves.

For completeness, we sketch here the argument for the proof of Proposition 3. The first step is to prove:

Lemma 1. If $X$ is a general Calabi-Yau hypersurface of dimension $\geq 2, X$ is not rationally swept out by elliptic curves of fixed modulus.

Proof. Indeed, fixing otherwise the modulus of the elliptic curve, we would get, for at least one elliptic curve $E$, an hypersurface $\mathcal{M}_{E}$ in the moduli space $\mathcal{M}$ of $X$ consisting of $X_{f}$ 's which are rationally dominated by some $E \times B$. For such an $X_{f}$, there must be an inclusion of rational Hodge structures induced by the dominant rational map $\phi: E \times B \rightarrow X_{f}$ :

$$
\phi^{*}: H^{n}\left(X_{f}\right)_{\text {prim }} \hookrightarrow H^{1}(E) \otimes H^{n-1}(B),
$$

because the Hodge structure on $H^{n}(X)_{\text {prim }}$ is simple for general $X$.

If we now let $f$ vary in $\mathcal{M}_{E}$, only $B$ deforms with $f$, not $E$, and it follows that the infinitesimal variation of Hodge structure on $H^{1}(E) \otimes H^{n-1}(B)$

$$
\bar{\nabla}: H^{p, q}\left(H^{1}(E) \otimes H^{n-1}(B)\right) \rightarrow H^{p-1, q+1}\left(H^{1}(E) \otimes H^{n-1}(B)\right) \otimes \Omega_{\mathcal{M}_{E}}
$$


has the following form at the point $f \in \mathcal{M}_{E}$ :

$$
\bar{\nabla}(\alpha \otimes \beta)=\alpha \otimes \bar{\nabla}_{B}(\beta)
$$

for $\alpha \in H^{r, s}(E), \beta \in H^{p-r, q-s}(B)$, where $\bar{\nabla}_{B}$ is the infinitesimal variation of Hodge structure on $H^{n-1}(B)$. Hence the Yukawa couplings of the IVHS on $H^{1}(E) \otimes H^{n-1}(B)$, that is the iterations of $\bar{\nabla}$, have the following property:

$$
\begin{aligned}
& \forall \eta \in H^{n, 0}\left(H^{1}(E) \otimes H^{n-1}(B)\right) \text {, the map } \\
& \qquad \bar{\nabla}^{n}(\eta): S^{n} T_{\mathcal{M}_{E}, f} \rightarrow H^{0, n}\left(H^{1}(E) \otimes H^{n-1}(B)\right)
\end{aligned}
$$

vanishes.

If there is along $\mathcal{M}_{E}$ an injective morphism of Hodge structures (3.19), it follows that the same property is true for the IVHS of the family of $X_{f}$ 's parameterized by $\mathcal{M}_{E}$, which implies that the Yukawa couplings of $X_{f}$ vanish on the hyperplane $K:=T_{\mathcal{M}_{E}, f} \subset S^{n+2}$. The Carlson-Griffiths theory [15], [2] shows easily that this is not the case. Indeed, these Yukawa couplings identify to the multiplication map

$$
S^{n}\left(S^{n+2}\right) \rightarrow R_{f}^{n(n+2)}
$$

Assume they vanish on $K$. Since $K$ is a hyperplane in $S^{n+2}$, the subspace

$$
K^{\prime}:=\left[K: S^{1}\right] \subset S^{n+1}
$$

has codimension $\leq n+2$. It is without base-point, since $T_{\mathcal{M}_{E}, f}$ contains $J_{f}$. It follows then from [13], that $S^{n+3} K^{\prime}=S^{2 n+4}$. But $K^{2}$ contains $S^{1} K^{\prime} \cdot K=$ $K^{\prime} \cdot S^{1} K=K^{\prime} \cdot S^{n+3}=S^{2 n+4}$. Hence $K^{2}=S^{2 n+4}$ and similarly $K^{n}=S^{n(n+2)}$ contradicting the fact that $K^{n} \subset J_{f}^{n(n+2)}$.

The second step is the following

LemMa 2. Assume a variety is rationally swept-out by elliptic curves, but not by elliptic curves with constant modulus. Then it admits a divisor which is rationally swept out by rational curves.

Proof. By assumption there is a diagram

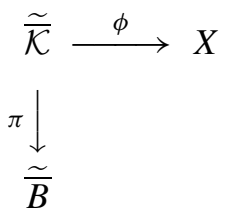

where we may assume that $\tilde{\bar{K}}$ and $\widetilde{\bar{B}}$ are smooth, projective, where $\tilde{\overline{\mathcal{K}}}$ is a smooth projective model of the family $\mathcal{K} \rightarrow B$ on which $\phi$ is defined, and that 
the map $j: \widetilde{\bar{B}} \rightarrow \mathbb{P}^{1}$ is defined and non constant. Now, since $\phi$ is generically finite, for generic $t \in \mathbb{P}^{1}$, the divisor $\widetilde{\overline{\mathcal{K}}}_{t}:=(j \circ \pi)^{-1}(t)$ must be sent by $\phi$ onto a divisor of $X$, and it follows that for any $t$ the image by $\phi$ of the divisor $\widetilde{\overline{\mathcal{K}}}_{t}:=(j \circ \pi)^{-1}(t)$ must contain a divisor of $X$. Taking $t=\infty$, and noting that any component of $\widetilde{\mathcal{K}}_{\infty}$ has a normalization which is uniruled, gives the result.

Proof of Theorem 3. In the proof of Theorem 1, we used first a countability argument for Chow varieties to show that if a dominating rational map

$$
\phi: K \rightarrow X
$$

exists for a general $X$, with $K$ fibred into varieties from the family $\mathcal{Y}$, there exists a universal dominating rational map

$$
\Phi: \mathcal{K} \rightarrow \mathcal{X}
$$

But the same argument can be applied as well to any codimension $n$ cycle in some $K \times X$, and even more generally to Hodge classes of degree $2 n$ on some $K \times X$. Indeed, the only point is to know that there are countably many quasi-projective varieties parameterizing the following data:

1. A $n$-dimensional variety $K$ which is fibred into varieties from the family $\mathcal{Y}$.

2. A smooth hypersurface $X_{f}$.

3. A dominating rational map from $K$ to $X_{f}$, or more generally a codimension $n$ cycle in $K \times X_{f}$, or even more generally a degree $2 n$ Hodge class $\gamma$ in $K \times X_{f}$, inducing a non-zero morphism of Hodge structures $H^{n}\left(X_{f}\right)_{\text {prim }} \rightarrow$ $H^{n}(K)$.

For the last case, one uses [3].

Furthermore, we need to know that on each quasi-projective variety as above there exists a universal object. In the case of Hodge classes, this is due to the global invariant cycle theorem of Deligne.

Next, at the end of the argument, we used the fact that the rational map $\phi_{f}: K_{f} \rightarrow X_{f}$ is dominating only to deduce that it induces inclusion

$$
\phi_{f}^{*}: H^{n}\left(X_{f}\right)_{\text {prim }} \rightarrow H^{n}\left(K_{f}\right)
$$

of Hodge structures.

Hence, the whole argument works in fact with "graphs of rational maps" replaced by "codimension $n$ algebraic cycles or degree $2 n$ Hodge classes in some product $K \times X$ inducing a non-zero morphism of Hodge structures $H^{n}(X)_{\text {prim }} \rightarrow$ $H^{n}(K)$ ", or more generally by "non-zero Hodge classes in $H^{n}(X)_{\text {prim }} \otimes H^{n}(K)$ ".

(Note that such a morphism, once it is assumed to be non-zero, would always have an image which is of Hodge level $n$, because the Hodge structure on $H^{n}(X)_{\text {prim }}$ is simple for generic $X$.)

Using the fact that the moduli space of curves of genus $g \geq 1$ has dimension $3 g-3, g \geq 2$ or $1, g=1$, we see that Theorem 3 is then a consequence of 
Theorem 1 , where the family $\mathcal{Y} \rightarrow \mathcal{S}$ is the family of curves of genus $g$, and where we replace as explained above dominating rational maps with degree $2 n$ non zero Hodge classes in $H^{n}(K) \otimes H^{n}\left(X_{f}\right)_{\text {prim }}$.

\section{REFERENCES}

[1] M. Asakura - S. SAito, Noether-Lefschetz locus for Beilinson-Hodge cycles on open complete intersections, preprint 2003.

[2] J. CARLSON - Рh. GRIffiths, Infinitesimal variations of Hodge structures and the global Torelli problem, In: "Journées de géométrie algébrique", A. Beauville (eds.), SijthoffNordhoff, 1980, pp. 51-76.

[3] E. Cattani - P. Deligne - A. Kaplan, On the locus of Hodge classes, J. Amer. Math. Soc. (2) 8 (1995), 483-506.

[4] L. Chiantini - A.-F. Lopez - Z. Ran, Subvarieties of generic hypersurfaces in any variety, Math. Proc. Cambr. Philos. Soc. (2002).

[5] H. Clemens, Curves in generic hypersurfaces, Ann. Sci. École Norm. Sup. 19 (1986), 629-636.

[6] H. Clemens, Curves on higher-dimensional complex projective manifolds, In: "Proceedings of the International Congress of Mathematicians", (2) 1 (Berkeley, Calif., 1986), 634-640.

[7] H. Clemens - J. Kollár - S. Mori, "Higher dimensional complex geometry", Astérisque 166, SMF (1988).

[8] H. Clemens - Z. Ran, Twisted genus bounds for subvarieties of generic hypersurfaces, Amer. J. Math. 126 (2004), 89-120.

[9] P. Deligne, Théorèmes de Lefschetz et critères de dégénérescence de suites spectrales, Publ. Math. Inst. Hautes Études Sci. 35 (1968), 107-126.

[10] P. Deligne, Théorie de Hodge II, Publ. Math. Inst. Hautes Études Sci. 40 (1971), 5-57.

[11] P. Deligne, La conjecture de Weil pour les surfaces K3, Invent. Math. 15 (1972), 206-226.

[12] L. Ein, Subvarieties of generic complete intersections, Invent. Math. 94 (1988), 163-169.

[13] M. GREEN, Restrictions of linear series to hyperplanes, and some results of Macaulay and Gotzmann, In: "Algebraic curves and projective geometry”, E. Ballico-C. Ciliberto (eds.), Lecture Notes in Mathematics 1389, Springer-Verlag 1989, pp. 76-86.

[14] M. GREEN, A new proof of the explicit Noether-Lefschetz theorem, J. Differential Geom. 27 (1988), 155-159.

[15] PH. GRIfFiths, Periods of certain rational integrals, I, II, Ann. of Math. 90 (1969), 460-541.

[16] S. Lang, Hyperbolic and diophantine Analysis, Bull. Amer. Math. Soc. (2) 14 (1986), 159-205.

[17] D. Mumford, Rational equivalence of zero-cycles on surfaces, J. Math. Kyoto Univ. 9 (1968), 195-204.

[18] M. NoRI, Algebraic cycles and Hodge theoretic connectivity, Invent. Math. 111 (1993), 349-373.

[19] A. Otwinowska, Asymptotic bounds for Nori's connectivity theorem, preprint 2002.

[20] C. Voisin, On a conjecture of Clemens on rational curves on hypersurfaces, J. Differential Geom. 44 (1996), 200-214, 49 (1998), 601-611. 
[21] C. Voisin, "Hodge Theory and Complex Algebraic Geometry II", Cambridge University Press, 2003.

[22] C. VoIsIn, Nori's connectivity theorem and higher Chow groups, J. Inst. Math. Jussieu (2) 1 (2002), 307-329.

Institut de mathématiques de Jussieu CNRS,UMR 7586 